\title{
Hacia un modelo jerárquico contextual y disposicional del aprendizaje*
}

\section{Towards a Contextual and Dispositional Hierarchical Model of Learning}

Recibido: 01 de febrero de 2015| Aceptado: 26 de marzo de 2016

\author{
José Antonio CeCChINI-EstradA ** \\ ANTONIO MÉNDEZ-GIMÉNEZ *** \\ JAVIER FERNÁNDEZ-RÍO ***** \\ Universidad de Oviedo, España
}

doi: 10.11144/Javeriana.upsy15-2.mjcd

Para citar este artículo: Cecchini-Estrada, J. A., Méndez-Giménez, A., \& Fernández-Río, J. (2016). Towards a contextual and dispositional hierarchical model of learning. Universitas Psychologica, 15(2), 217-230. http://dx.doi.org/10.11144/Javeriana. upsy15-2.mjcd

* Artículo de investigación científica y tecnológica. Esta investigación se ha realizado en el marco del proyecto de I+D+I número DEP2012-31997, subvencionado por el Ministerio de Economía y Competitividad.

** Catedrático de Universidad. Departamento Ciencias de la Educación. Correo electrónico: cecchini@uniovi.es

*** Titular de Universidad interino. Departamento Ciencias de la Educación. Correo electrónico: mendezantonio@uniovi.es

******* Titular de Universidad. Departamento Ciencias de la Educación. Correo electrónico: javier.rio@ uniovi.es

\section{RES UMEN}

El propósito de la investigación es examinar el patrón secuencial de un modelo teórico que incluye variables contextuales y disposicionales en el aprendizaje de tres materias de educación secundaria obligatoria: Matemáticas (MT), Lengua Castellana y Literatura (LC) y Educación Física (EF). La muestra estuvo formada por 1108 estudiantes de educación secundaria (374 de MT, 364 de LC y 370 de EF). Se constató la validez del modelo mediante un análisis de estructuras de covarianzas, primero de manera independiente en cada asignatura, enfrentándolo, además, con modelos rivales. De los modelos testados el que presenta mejores valores, en los tres casos, es el que se hipotetizó en este estudio, con resultados muy similares en todos los parámetros estimados a los observados en el análisis factorial confirmatorio preliminar. El análisis multistep de invarianza mostró que los modelos presentaban índices de ajuste aceptables. El modelo de estructura de covarianza testado sitúa al alumnado en el eje de todo el proceso y al profesor como un ayudante eficaz, además permite superar la tradicional contraposición entre esfuerzo e interés.

Palabras clave

matemáticas; educación física; lengua; educación secundaria; cultura del esfuerzo; interés

\section{A B S T R A C T}

The purpose of this research is to examine the sequential pattern of a theoretical model that includes contextual and dispositional variables in the learning of three Secondary Education subjects: Maths (MT), Spanish Language and Literature (LC) and Physical Education (PE). The sample consisted of 1108 students of Secondary Education $(\mathrm{MT}=374, \mathrm{LC}=364$, and $E F=370$ ). The validity of the model was verified by analysis of covariance structures, first independently in each subject, also facing it with rival models. The hypothesized model tested in this study yielded the best values in all three cases, with very similar results in all estimated parameters to those observed in the preliminary confirmatory factor analysis. Invariance multistep analysis showed that the models had acceptable fit indices. The tested covariance structure model places the student at the center of the whole process, and the teacher as an effective assistant. It allows us to overcome the traditional opposition between effort and interest.

Keywords

Maths; Physical Education; Language; Secondary Education; Culture of effort; Interest 
El fracaso escolar es un problema grave de nuestro sistema educativo (Colectivo Lorenzo Luzuriaga, 2012). De manera consistente, los Estados miembros de la Unión Europea han reconocido que el abandono prematuro de la escuela representa un problema importante para las sociedades y las personas. Según el Informe de seguimiento de la educación para todos en el mundo (Unesco, 2012), en España uno de cada tres jóvenes no termina el segundo ciclo de la enseñanza secundaria, lo que arrastrará consecuencias negativas para los propios estudiantes, sus familias y la sociedad en su conjunto.

Algunos autores defienden que el esfuerzo, o más concretamente la ausencia del mismo, es una de las causas principales del fracaso escolar (Del Burgo, 2002; Fernández, Mena, \& Riviere, 2010; Marchesi, 2003). Este factor es relevante y explica una parte de los problemas del aprendizaje. Sin embargo, esta realidad no debería analizarse de forma aislada e independiente del interés por el estudio y de los condicionamientos educativos familiares y sociales (Lozano, 2003). Cada vez es mayor el número de personas que, desde diferentes ámbitos, reclaman al sistema educativo un retorno de "cultura del esfuerzo" (Cecchini, Fernández-Losa, \& Cecchini, 2013). Según los últimos informes internacionales, los estudiantes finlandeses obtienen los mejores resultados académicos del mundo y lo consiguen con "poca inversión" y "mucho esfuerzo" (Enkvist, 2012). Esta autora critica duramente la pedagogía del interés que establece, entre otras cuestiones, que todo aprendizaje debe basarse en el propio alumno, en sus conocimientos anteriores y en sus centros de intereses. De esta posición se ha extraído la idea de que el alumno es y debe ser su propia autoridad en materia de aprendizaje y que debe gustarle aprender. El método se ha basado en pedir al docente que sea capaz de crear en el alumnado un interés por el aprendizaje más que en exigirle su propio esfuerzo (Enkvist, 2012). En desacuerdo con estos planteamientos, esta autora propone volver a una "pedagogía del esfuerzo" en la que la voluntad y la laboriosidad sirvan para vencer las dificultades que se derivan del proceso de aprender. Además, plantea un divorcio, a veces insuperable, entre el interés del alumnado por el aprendizaje y el esfuerzo a invertir en el mismo.
En este sentido, la pregunta de por qué los estudiantes no se esfuerzan suficientemente en clase está latente entre los padres, docentes e investigadores. Diferentes estudios han informado de que alrededor del 50\% de los estudiantes manifiesta que sus clases son aburridas (Shernoff, Csikszentmihalyi, Schneider, \& Shernoff, 2003). Más allá, Hektner, Schmidt y Csikszentmihalyi (2007) observaron que cuando los adolescentes estaban en clase se sentían generalmente más aburridos, irritables y tristes en comparación con la experiencia afectiva que tenían en otros contextos de su vida. Esta variable disposicional del aprendizaje parece no estar tan relacionada con la habilidad para aprender como por la calidad de las experiencias académicas (Schiefele \& Csikszentmihalyi, 1995). La falta de contenidos vitales y actuales para el alumnado (Roca et al., 2010), la exigua autonomía percibida (Wong, 2000), el escaso poder de participación (Yair, 2000) o la monotonía de las tareas de enseñanza (Larson \& Richards, 1991), entre otras, son algunas de las causas que explican la falta de compromiso del alumnado.

Según Schank (2005) una de las labores asignadas al profesor es la de despertar el interés de los jóvenes por el estudio, el enseñar a aprender. Por este motivo, en estos últimos años se han propuesto modelos de enseñanza-aprendizaje cuya finalidad es despertar el interés de los estudiantes hacia el aprendizaje en la enseñanza de las diversas materias: Economía (e. g., Carroza, Alcaide, Heredia, Humanes, \& Velasco, 2008), Geografía e Historia (e. g., Cuenca, 2010), Física (e. g., Häusemann, 2011), Química (e. g., Franco, 2012), Matemáticas (e. g., Mendoza, 2008; Sabater, 2009), Lengua Española (e. g., Centelles, 2012), Educación Física (e. g., Méndez-Giménez, 2011) o Música (e. g., Flores, 2011).

La relación entre el esfuerzo y el interés en el proceso formativo sigue siendo, en nuestros días, un asunto sin resolver. ¿Qué está ocurriendo en la actualidad en las aulas españolas en lo referente a estas cuestiones? ¿Cómo se está desarrollando la labor docente y qué incidencia está teniendo en el aprendizaje? Para tratar de dar respuesta a estas preguntas, Cecchini, González-Pienda et al. (2014) elaboraron y validaron dos escalas que pretenden 
medir determinadas variables contextuales y disposicionales relacionadas con el aprendizaje en el alumnado de educación secundaria, y que, además, se pueden aplicar en diversas materias del currículum. A la primera la denominaron IEPA y está formada por las siguientes subescalas: a) interés por aprender, b) progresión en el aprendizaje y c) esfuerzo para aprender. A la segunda la denominaron AYES, y está formada por otras tres subescalas: a) despertar el interés hacia el aprendizaje, b) ayudar a aprender y c) promover una cultura del esfuerzo.

Estos instrumentos se brindan como herramientas para analizar la vieja contraposición entre la "pedagogía del interés" y la "pedagogía del esfuerzo". Pueden ser útiles para que los investigadores desvelen lo que está sucediendo en la actualidad en los colegios y, en base a los resultados, aclaren lo que debería ocurrir para promover un aprendizaje eficaz. Cecchini, González-Pienda et al. (2014) sugieren que en la práctica educativa cotidiana estas dos dimensiones, para algunos tan distantes, no sólo no están enfrentadas sino que se complementan, de tal manera que para ser un docente eficaz hay que ser capaz no solo de despertar el interés de los alumnos por la materia, sino también de promover una cultura del esfuerzo. Ambas dimensiones del aprendizaje, en opinión de estos autores, parecen estar fuertemente relacionadas. Desde los paradigmas contemporáneos, el profesor eficaz es aquel que sitúa a sus alumnos en el centro de todo el proceso educativo y se convierte a sí mismo en un mediador entre el alumno y la materia; el que enseña a aprender a aprender, a tomar conciencia de lo que están aprendiendo y de cómo lo están aprendiendo, y a regular los procesos que les permitan seguir aprendiendo en un futuro. Para conseguir todos estos objetivos, es preciso que el profesor modifique su forma de enseñar y consiga que el alumno se implique en actividades sugestivas en las que encuentre algún tipo de relación con su vida diaria (Marchesi, 2003).

Por estos motivos, la tradicional contraposición entre esfuerzo e interés, en nuestros días, parece cuestionable. Esfuerzo e interés son dos variables que, por un lado, podrían incidir por separado en el aprendizaje (y por tanto, complementarse), y, por otro lado, podrían interaccionar entre sí en el proceso de aprendizaje (y, por tanto, interactuar). Lejos de hablar de contraposición entre interés y esfuerzo, quizás se debería hablar de interdependencia y, en consecuencia, de colaboración entre ambas. Por ello, promover una "cultura del esfuerzo" en las clases debería ir unido a una acción docente capaz de despertar en el alumnado el interés por la materia y la diversión en el aprendizaje, porque todo ello podría estar fuertemente relacionado. Por un lado, el aprendizaje puede ser vivenciado por el alumnado como algo interesante y sugestivo, que responde seguramente a una necesidad vital que le lleva a participar en el entorno físico y social y, a asimilar los valores y prácticas ambientales y culturales. Este gusto por el conocimiento puede no ir asociado a sentimientos de esfuerzo, entendido éste como abnegación, entrega o sacrificio. El esfuerzo, como sinónimo de sacrificio, en la educación tiene sentido cuando aprender no resulta divertido. El sacrificio sería necesario, en este caso, para conseguir un objetivo valioso que no se puede alcanzar de otro modo, es decir, el esfuerzo al margen del interés por el aprendizaje. Pero, además, el esfuerzo puede ser la consecuencia del placer de aprender, en este caso el esfuerzo intermedia entre el interés del alumno y el progreso en el aprendizaje y tiene un significado muy distinto para él, ya que lo vivencia de una manera positiva. Aunque el aprendizaje a menudo requiere esfuerzo, éste no tiene porqué ser incompatible con el gozo, como no lo es en el jugador de ajedrez o en el aficionado al alpinismo (Trilla, 2002). Es posible, por tanto, que esfuerzo, interés y aprendizaje sean variables muy interrelacionadas y que determinados alumnos lleguen al aprendizaje fundamentalmente a través del esfuerzo, otros lo hagan prioritariamente a través del interés y, otros, de manera conjunta.

En esta investigación se propone un modelo contextual y situacional del aprendizaje con base en los siguientes principios: 1 . El alumno es un sujeto activo, que como tal, se hace cargo de su propio aprendizaje; 2. El docente deber ser un mediador eficaz en el aprendizaje de sus alumnos: a) ayudándoles a aprender, b) despertando su interés por la materia y c) promoviendo una cultura del esfuerzo; 
3. Estas variables contextuales influyen en las variables disposicionales del aprendiz, es decir, en la progresión en el aprendizaje, en el interés que despierta hacia la materia y en el esfuerzo invertido para aprender; 4. Estas variables también representan niveles de eficacia, pero en este caso del alumno, de tal forma que el aprendizaje depende de su interés por conseguir metas de aprendizaje, de su esfuerzo para alcanzarlas y de su capacidad para autorregular el aprendizaje. Se refieren a aprendices que buscan retos activamente y que superan los obstáculos, a veces con gusto y creatividad para resolver problemas y, otras, con persistencia y esfuerzo para superar esas dificultades, y las más de las veces con una combinación de ambos: un esfuerzo consecuencia del interés por los retos que se le proponen.

La finalidad de este estudio es elaborar y validar un modelo jerárquico contextual y situacional del aprendizaje. Para ello, se plantea un modelo de ecuaciones estructurales en el que se espera que las variables contextuales del aprendizaje estén fuertemente relacionadas y que, por tanto sus covarianzas $(\Phi)$ sean elevadas, ya que todas ellas son dimensiones de la ayuda al aprendizaje; de hecho, se cree que muestran niveles de eficacia en la acción docente. También se hipotetizó que cada una de estas variables contextuales predeciría una variable disposicional $(\gamma)$, por lo que se espera encontrar un paso significativo entre la acción docente destinada a despertar el interés del alumnado por la materia de enseñanza y la diversión del alumno en el aprendizaje, otro paso entre la actividad del profesor dirigida a resolver los problemas encontrados en el proceso de aprendizaje de la materia con la mejora y la adquisición de habilidades específicas por parte del alumnado, y, por último, otro paso entre el reconocimiento, la valoración y la recompensa de la participación y el esfuerzo del alumno en el aprendizaje y el esfuerzo empleado por éste para alcanzar los objetivos de la materia. Teniendo en cuenta que el elemento central de todo el proceso es el alumno, también se espera encontrar relaciones directas $(\beta)$ entre el interés que en él despierta la materia y la adquisición de habilidades o competencias y entre el esfuerzo invertido por el alumno, además de la adquisición de habilidades o competencias. Asi- mismo, se hipotetizó una relación indirecta entre el "interés en el aprendizaje" con la "progresión en el aprendizaje" a través del "esfuerzo en el aprendizaje", de tal modo que el interés del alumnado por la materia predice su nivel de esfuerzo, que sucesivamente predice el aprendizaje (figura 1).

\section{Método}

\section{Muestra}

La muestra estuvo formada por 1108 estudiantes adolescentes españoles de diez institutos de educación secundaria (de $1^{\mathrm{O}}$ a $4^{\mathrm{O}}$ de ESO), con edades comprendidas entre los 12 y 16 años, seleccionados al azar. Los análisis se realizaron en tres conjuntos de datos relacionados con tres materias: Matemáticas (MT, $\mathrm{n}=374$, con un media de edad $=13.55 \pm$ 1.14 años); Lengua Castellana y Literatura (LC, $\mathrm{n}=$ 364 , con un media de edad $=13.53 \pm 1.04$ años), y Educación Física (EF, $n=370$, con un media de edad $=13.71 \pm 1.12$ ). Los datos para todos los estudios se recogieron inmediatamente después de la sesión correspondiente a cada materia estudiada. La participación en todos los estudios fue voluntaria.

\section{Instrumentos}

\section{Cuestionario de interés, esfuerzo y progreso en el aprendizaje (IEPA)}

El primero de los cuestionarios utilizados se denomina IEPA (Interés, esfuerzo y progreso en el aprendizaje) y ha sido elaborado y validado por Cecchini, González-Pienda et al. (2014) para su uso en las materias de Matemáticas, Lengua Castellana y Literatura, y Educación Física en educación secundaria. Su finalidad, es evaluar tres variables disposicionales del alumnado en el aprendizaje. Cada subescala está formada por cuatro ítems: el interés en el aprendizaje (e.g. "el aprendizaje es interesante y entretenido"), el esfuerzo en el aprendizaje (e.g. "me esfuerzo por aprender") y la progresión en el aprendizaje (e.g. "veo que estoy mejorando"). Las preguntas van precedidas de la introducción "en las clases de (Matemáticas, Lengua Castellana y Litera- 


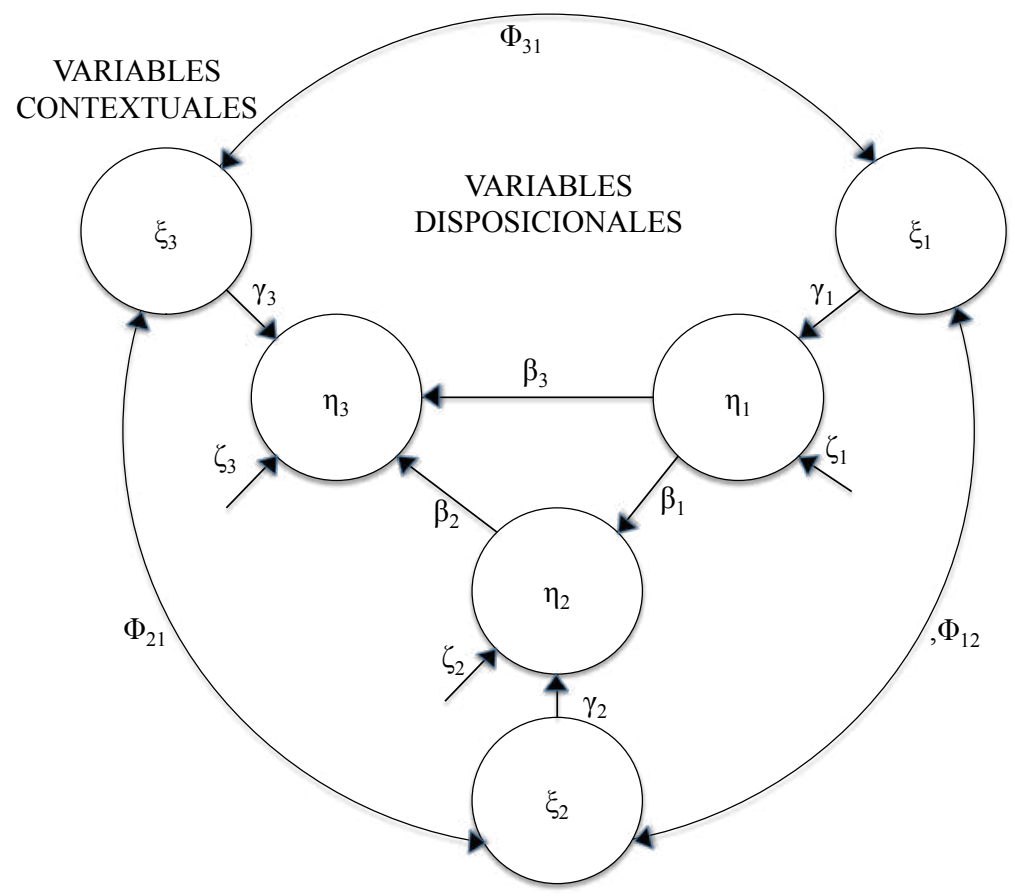

Figura 1. Variables latentes exógenas: despertar el interés del alumno por el aprendizaje $\left(\xi_{1}\right)$, promover el valor del esfuerzo $\left(\xi_{2}\right)$, y ayudar a aprender $\left(\xi_{3}\right)$. Variables latentes endógenas: interés en el aprendizaje $\left(\eta_{1}\right)$, esfuerzo en el aprendizaje $\left(\eta_{2}\right)$, y progresión en el aprendizaje $\left(\eta_{3}\right)$. Parámetros estructurales $(\gamma)$. Parámetros entre variables latentes endógenas $(\beta)$. Covarianzas $(\Phi)$. Errores de regresión ( $(\zeta)$.

Fuente: elaboración propia.

tura, Educación Física)...”. Los sujetos responden en una escala tipo Likert de 5 puntos que oscila desde "muy de acuerdo" (5) a "muy en desacuerdo" (1). Las distintas subescalas han mostrado unos adecuados índices de consistencia interna (alfa de Cronbach) entre 0.81 y 0.93 .

\section{Cuestionario de ayuda al estudiante (AYES)}

La segunda de las escalas utilizadas se denomina AYES (Ayuda al estudiante) y su objetivo es medir tres variables contextuales relacionadas con la percepción del alumnado sobre la acción docente destinada a conseguir los siguientes objetivos: a) despertar el interés del alumno por el aprendizaje (e.g. "las tareas y lecciones despiertan el interés de los alumnos"), b) ayudar a aprender (e.g. "el profesor sabe cómo ayudarnos y lo hace"), y c) promover el valor del esfuerzo (e.g. "se valora la participación y el esfuerzo de los estudiantes"). Cada subescala también está formada por cuatro ítems. Esta escala también ha sido elaborada y validada por Cecchini, González-Pienda et al. (2014) y Cecchini, MéndezGiménez, \& Fernández-Río (2014) en las materias de Matemáticas, Lengua Castellana y Literatura, y Educación Física para educación secundaria. Las preguntas van precedidas de la introducción: "en las clases de (Matemáticas, Lengua Castellana y Literatura, Educación Física)...”. Se utilizó una escala tipo Likert de 5 puntos que oscila desde "muy de acuerdo" (5) a "muy en desacuerdo" (1). Las distintas subescalas mostraron adecuados índices de consistencia interna (alfa de Cronbach entre 0.86 y 0.91$)$.

\section{Procedimiento}

Se obtuvo el consentimiento informado de los padres y directores de los centros. Se comentó a los estudiantes que los cuestionarios eran anónimos y se les 
aseguró que sus respuestas no estarían a disposición de sus maestros o padres. También se les ofreció la opción de rechazar la participación o retirarse en cualquier momento. Todos los cuestionarios fueron completados bajo la supervisión de un investigador experimentado. La duración aproximada de la cumplimentación de los cuestionarios fue de 25 minutos.

\section{Resultados}

\section{Análisis descriptivos, alfas de Cronbach y correlaciones bivariadas}

En la tabla 1 se presentan los estadísticos descriptivos de cada una de las variables de estudio. La materia de Educación Física es la que presenta valores más altos en todas las variables analizadas, salvo en "ayuda al aprendizaje" que puntuó por detrás de Matemáticas. En las tres materias los valores más altos se observan en el factor "esfuerzo por aprender", salvo en Matemáticas que va detrás de "ayudar al aprendizaje", y la más baja en "despertar el interés hacia el aprendizaje".

En el análisis de correlaciones se observa que todas las variables se relacionan de forma significativa entre sí $(p<0.001)$. Los alfas de Cronbach oscilan entre 0.79 y 0.93 (tabla 2), estando todos por encima del valor recomendado: 0.70 (Nunnally, 1978).

\section{Analisis de ecuaciones estructurales}

A pesar de que se partía de un modelo teórico (figura 1), se decidió seguir las indicaciones de Lévy,
Martín, \& Roman (2006) y el procedimiento en dos pasos recomendado por Anderson \& Gerbing (1988). En primer lugar, se procedió a evaluar la validez del constructo mediante un análisis factorial confirmatorio (AFC), teniendo en cuenta las veinticuatro medidas observadas y los seis constructos latentes que correlacionaban libremente. A continuación, como no se había comprobado en la teoría la existencia de un modelo adecuado, planteamos modelos rivales, pues un modelo puede ajustarse bien a los datos, pero no por eso poseer las propiedades de la generalización (Lévy et al., 2006). El modelo 1 es un modelo causal con un nivel de causalidad, en el que los factores disposicionales (variables latentes exógenas) covarían entre sí y a su vez cada uno de ellos predice una variable latente endógena. Por ello, se incluyó un paso entre el factor "despertar el interés hacia el aprendizaje" e "interés por aprender", otro entre "ayudar en el aprendizaje" y "progresión en el aprendizaje", y un tercero entre "promover una cultura del esfuerzo" y "esfuerzo para aprender". El modelo 2 es un modelo causal con dos niveles de causalidad, en el que además de los pasos anteriores, relacionamos dos variables latentes endógenas con una tercera. Para ello, se incluyeron dos pasos, uno desde "interés por aprender" y otro desde "esfuerzo para aprender" a "progresión en el aprendizaje". El modelo 3 contiene, además, efectos causales indirectos entre variables latentes exógenas, por lo que se añadió un paso más desde "interés por aprender" a "esfuerzo para aprender". Los modelos se examinaron en las respuestas dadas por los estudiantes a las cuestiones

TABLA 1.

Media y desviación típica para las tres materias

\begin{tabular}{lcccccc}
\hline \multirow{2}{*}{ Factores } & \multicolumn{2}{c}{ Matemáticas } & \multicolumn{2}{c}{ Lengua Castellana } & \multicolumn{2}{c}{ Educación Física } \\
& $\mathrm{M}$ & $\mathrm{DT}$ & $\mathrm{M}$ & $\mathrm{DT}$ & $\mathrm{M}$ & $\mathrm{DT}$ \\
\hline 1. Despertar el interés hacia el aprendizaje & 2.86 & 0.94 & 2.78 & 0.91 & 3.66 & 0.88 \\
2. Ayudar en el aprendizaje & 3.89 & 0.93 & 3.66 & 1.04 & 3.86 & 0.94 \\
3. Promover una cultura del esfuerzo & 3.67 & 0.97 & 3.50 & 1.10 & 3.82 & 1.04 \\
4. Interés por aprender & 2.89 & 0.93 & 2.85 & 0.94 & 3.92 & 0.85 \\
5. Progresión en el aprendizaje & 3.74 & 0.89 & 3.64 & 0.88 & 3.80 & 0.84 \\
6. Esfuerzo para aprender & 3.84 & 0.80 & 3.85 & 0.79 & 4.08 & 0.71 \\
\hline
\end{tabular}

Fuente: Elaboración propia 
TABLA 2.

Correlaciones entre todas las variables en cada materia por separado

\begin{tabular}{|c|c|c|c|c|c|c|}
\hline \multirow[b]{2}{*}{ Factores } & \multicolumn{6}{|c|}{ Matemáticas } \\
\hline & 1 & 2 & 3 & 4 & 5 & 6 \\
\hline 1. Despertar el interés hacia el aprendizaje & 0.88 & & & & & \\
\hline 2. Ayudar en el aprendizaje & $0.55 * *$ & 0.90 & & & & \\
\hline 3. Promover una cultura del esfuerzo & $0.50 * *$ & $0.68 * *$ & 0.92 & & & \\
\hline 4. Interés por aprender & $0.73 * *$ & $0.43 * *$ & $0.37 * *$ & 0.90 & & \\
\hline 5. Progresión en el aprendizaje & $0.46 * *$ & $0.46 * *$ & $0.44 * *$ & $0.47 * *$ & 0.92 & \\
\hline \multirow[t]{2}{*}{ 6. Esfuerzo para aprender } & $0.39 * *$ & $0.41 * *$ & $0.38 * *$ & $0.51 * *$ & $0.55 * *$ & 0.85 \\
\hline & \multicolumn{6}{|c|}{ Lengua Castellana y Literatura } \\
\hline Factores & 1 & 2 & 3 & 4 & 5 & 6 \\
\hline 1. Despertar el interés hacia el aprendizaje & 0.88 & & & & & \\
\hline 2. Ayudar en el aprendizaje & $0.58 * *$ & 0.91 & & & & \\
\hline 3. Promover una cultura del esfuerzo & $0.55 * *$ & $0.71 * *$ & 0.93 & & & \\
\hline 4. Interés por aprender & $0.72 * *$ & $0.41 * *$ & $0.41 * *$ & 0.89 & & \\
\hline 5. Progresión en el aprendizaje & $0.46 * *$ & $0.50 * *$ & $0.46 * *$ & $0.57 * *$ & 0.92 & \\
\hline \multirow[t]{2}{*}{ 6. Esfuerzo para aprender } & $0.40 * *$ & $0.30 * *$ & $0.35 * *$ & $0.41 * *$ & $0.46 * *$ & 0.85 \\
\hline & \multicolumn{6}{|c|}{ Educación Física } \\
\hline Factores & 1 & 2 & 3 & 4 & 5 & 6 \\
\hline 1. Despertar el interés hacia el aprendizaje & 0.86 & & & & & \\
\hline 2. Ayudar en el aprendizaje & $0.61 * *$ & 0.88 & & & & \\
\hline 3. Promover una cultura del esfuerzo & $0.59 * *$ & $0.70 * *$ & 0.92 & & & \\
\hline 4. Interés por aprender & $0.70 * *$ & $0.43 * *$ & $0.44 * *$ & 0.87 & & \\
\hline 5. Progresión en el aprendizaje & $0.49 * *$ & $0.44 * *$ & $0.36 * *$ & $0.54 * *$ & 0.90 & \\
\hline 6. Esfuerzo para aprender & $0.46^{* *}$ & $0.41 * *$ & $0.39 * *$ & $0.51 * *$ & $0.53 * *$ & 0.79 \\
\hline
\end{tabular}

Nota: en la diagonal se incluyen los alfas de Cronbach. $* * p<0.01$

Fuente: elaboración propia.

que se les plantearon en cada materia de manera independiente (Matemáticas, Lengua Castellana y Literatura y Educación Física). El objetivo de este análisis preliminar es, también, demostrar que el modelo de base especificado presenta un buen ajuste en todas las muestras y es perfectamente interpretable en cualquiera de ellas (Abalo, Lévy, Rial, \& Varela, 2006).

Todos los análisis se realizaron utilizando el programa EQS 6.2 (Bentler, 2006). Dado que el análisis previo de los datos en todos los modelos reveló una curtosis multivariante sustancial, se realizó un análisis que se basa en la utilización del estadístico Satorra-Bentler chi-cuadrado ( $\mathrm{S}-\mathrm{B} \chi^{2}$; Satorra \& Bentler, 1994) y de los estimadores estándar robustos implementados en el programa estadístico EQS, en lugar del habitual estadístico de máxima verosimilitud chi-cuadrado (ML $\chi^{2}$ ), ya que sirve como corrección para $\chi^{2}$ cuando las suposiciones de distribución son violadas. Con demasiada frecuencia, los investigadores que deseen realizar los análisis SEM parecen no estar bien informados con respecto a los conceptos y cuestiones relacionadas con la asunción crítica de normalidad multivariante (Byrne, 2008). La investigación ha demostrado que la curtosis afecta gravemente a las pruebas de varianzas y covarianzas. En particular, ahora se sabe que la curtosis multivariante es excepcionalmente perjudicial para la estimación de parámetros en el análisis SEM (e.g., Byrne, 2008).

En cada uno los modelos descritos anteriormente, la evaluación de la bondad del ajuste de los datos se determinó sobre la base de criterios múltiples (Byrne, 2008): como índices de ajuste incre- 
TABLA 3.

Adecuación de los modelos propuestos a los datos

\begin{tabular}{ccccccccc}
\hline & Modelo & S-B $\chi 2$ & S-B $\Delta \chi 2$ & df & *CFI & \multicolumn{2}{c}{ SRMR } & *RMSEA (90\% CI) \\
\hline \multirow{6}{*}{ MT } & AFC & 339.24 & - & 237 & 0.98 & 0.04 & 0.034 & $(0.025-0.042)$ \\
& M1 & 476.13 & 136.89 & 246 & 0.96 & 0.12 & 0.050 & $(0.043-0.057)$ \\
& M2 & 404.69 & 65.45 & 244 & 0.97 & 0.09 & 0.042 & $(0.035-0.049)$ \\
& M3 & 352.95 & 13.71 & 243 & 0.98 & 0.04 & 0.035 & $(0.027-0.043)$ \\
& AFC & 341.16 & - & 237 & 0.98 & 0.04 & 0.035 & $(0.026-0.043)$ \\
& M1 & 456.40 & 115.24 & 246 & 0.96 & 0.10 & 0.049 & $(0.041-0.055)$ \\
& M2 & 384.45 & 43.29 & 244 & 0.97 & 0.07 & 0.040 & $(0.032-0.047)$ \\
& M3 & 356.03 & 14.87 & 243 & 0.98 & 0.04 & 0.036 & $(0.027-0.043)$ \\
\hline EF & AFC & 321.72 & - & 237 & 0.98 & 0.04 & 0.031 & $(0.022-0.039)$ \\
& M1 & 447.13 & 125.41 & 246 & 0.95 & 0.11 & 0.047 & $(0.040-0.054)$ \\
& M2 & 376.43 & 54.71 & 244 & 0.96 & 0.08 & 0.038 & $(0.030-0.046)$ \\
& M3 & 333.50 & 11.78 & 243 & 0.98 & 0.04 & 0.031 & $(0.022-0.039)$ \\
\hline
\end{tabular}

Fuente: elaboración propia.

mental se empleó el *CFI (Comparative Fit Index), como medida de los índices de ajuste absoluto que determinan el grado en que el modelo predice la matriz de covarianza se utilizó también el *RMSEA (Root Mean Square Error Aproximation) y el SRMR (Root Mean Square Residual). El *CFI representa la versión robusta del CFI que se calcula en base al estadístico S-B $\chi^{2}$. Hu y Bentler (1999) sugieren un valor de 0.95 como indicativo de buen ajuste. El *RMSEA es una versión robusta del usual RMSEA y tiene en cuenta el error de aproximación en la población. Esta discrepancia se expresa por cada grado

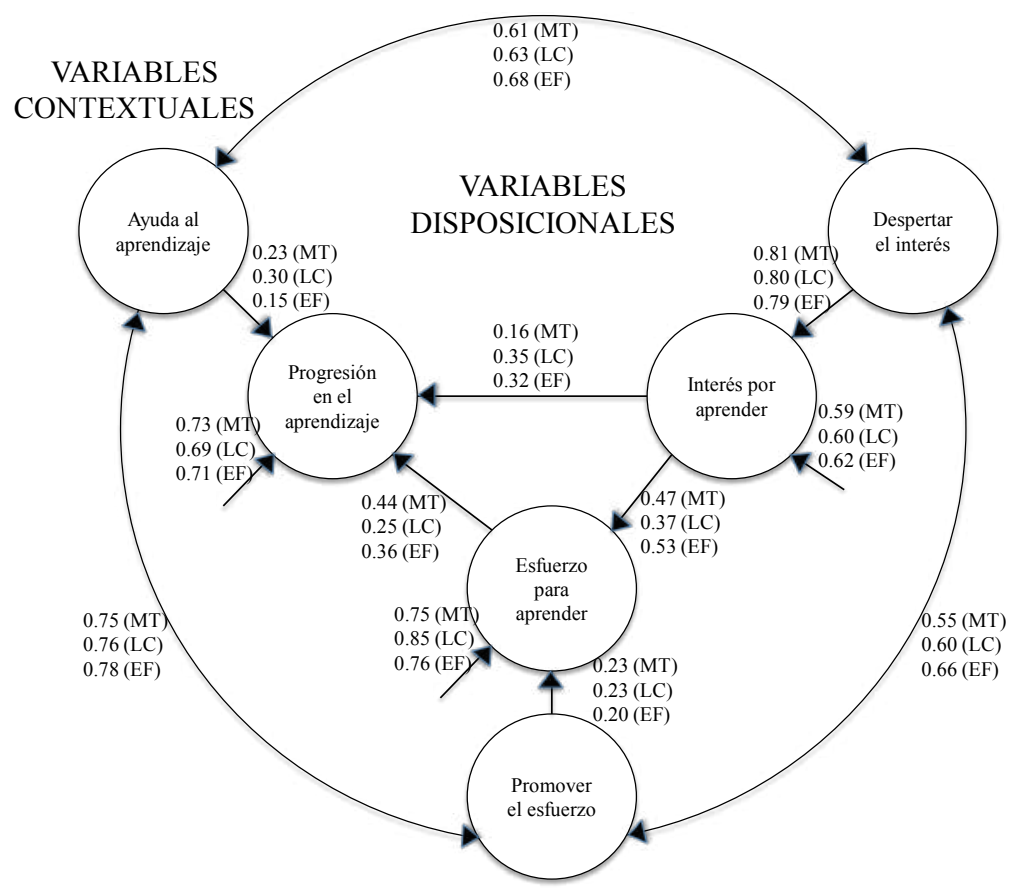

Figura 2. Análisis de ecuaciones estructurales del modelo 3, en las materias de Matemáticas (MT), Lengua Castellana y Literatura (LC) y Educación Física (EF), por separado

Fuente: elaboración propia. 
de libertad, por lo que es sensible a la complejidad del modelo, los valores inferiores a 0.05 indican un buen ajuste, y valores tan altos como 0.08 representan errores razonables de aproximación. Para completar el análisis, también se incluyó el intervalo de confianza al $90 \%$ proporcionado por *RMSEA (Steiger, 1990). Por último, la SRMR con un valor inferior a 0.08 es indicativa de un buen ajuste $(\mathrm{Hu}$ $\&$ Bentler, 1999). Los resultados pueden observarse en la tabla 3.

De todos los modelos testados, el que presenta mejores valores es el modelo 3 en todas las materias, con resultados muy similares a los observados en el análisis preliminar (AFC), por lo que no existe posibilidad de mejora (figura 2).

\section{Análisis factorial confirmatorio multimuestra e invarianza factorial}

El buen ajuste alcanzado en cada materia por separado no puede ser interpretado como que la estructura factorial es estrictamente invariante entre los grupos, ya que la estimación de los parámetros podría arrojar valores significativamente diferentes en cada uno de ellos (Lévy et al., 2006). A fin de generalizar y poner a prueba de forma más estricta el modelo hipotetizado, se analizó la invarianza factorial. Para examinar si los parámetros del modelo se mantuvieron invariantes a través de las tres materias, se empleó un análisis multistep de invarianza (Byrne, 2008; Marsh, 1993). El primer paso consiste en analizar el modelo inicial sin restricciones, ya que proporciona una base fundamental para las posteriores comparaciones de los modelos (Marsh, 1993). A continuación, obligamos a los pesos de medida a permanecer invariantes. El siguiente paso consiste en limitar los pesos estructurales estando los pesos de medida también limitados. El cuarto paso obliga, además, a que las covarianzas permanezcan estables. El penúltimo paso implica restringir también las varianzas factoriales. Por último, los errores de medida también deben permanecer invariantes.

Los resultados (tabla 4) mostraron que los modelos comparados presentaban índices de ajuste aceptables. Dado que el coeficiente $\chi^{2}$ es sensible al tamaño de la muestra, se empleó el criterio establecido por Cheung $\&$ Rensvold (2002) respecto al $\Delta^{*} \mathrm{CFI}$. Según estos autores, valores de $\Delta^{*} \mathrm{CFI}$ inferiores o iguales a -0.01 indican que no se puede rechazar la hipótesis nula de la invarianza. Los valores de $\Delta^{*} \mathrm{CFI}$ encontrados en este estudio en la comparación del modelo sin restricciones con los modelos con invarianza sugieren que la estructura factorial de ambos cuestionarios es en gran medida invariante en las tres muestras analizadas.

\section{Discusión}

El propósito de este estudio fue examinar el patrón secuencial de un modelo teórico que incluye variables contextuales y disposicionales en el aprendizaje de tres materias de la educación secundaria obligatoria, en principio, muy diferentes entre sí por las características específicas de la materia y sus contenidos

\section{TABla 4.}

Comparación del modelo hipotético con modelos alternativos

\begin{tabular}{ccccccccc}
\hline Modelo & S-B $\chi 2$ & $\mathrm{df}$ & $\mathrm{S}-\mathrm{B} \Delta \chi^{2}$ & $\Delta \mathrm{gl}$ & $*$ CFI & \multicolumn{2}{c}{ SRMR } & \multicolumn{2}{c}{$*$ RMSEA $(90 \%$ CI $)$} \\
\hline M1 & 1038.96 & 729 & - & - & 0.98 & 0.05 & 0.034 & $(0.029-0.038)$ \\
M2 & 1084.65 & 765 & 45.69 & 36 & 0.98 & 0.05 & 0.034 & $(0.029-0.038)$ \\
M3 & 1099.90 & 777 & 15.25 & 12 & 0.98 & 0.05 & 0.034 & $(0.029-0.038)$ \\
M4 & 1106.11 & 783 & 6.21 & 6 & 0.98 & 0.05 & 0.034 & $(0.029-0.038)$ \\
M5 & 1117.74 & 789 & 11.63 & 6 & 0.98 & 0.05 & 0.034 & $(0.029-0.038)$ \\
M6 & 1182.46 & 843 & 64.72 & 54 & 0.98 & 0.05 & 0.034 & $(0.029-0.038)$ \\
\hline
\end{tabular}

Nota: modelo 1 = sin restricciones; modelo 2 = pesos de medida invariantes; modelo 3 = pesos estructurales invariantes; modelo 4 = covarianzas estructurales invariantes; modelo $5=$ varianzas factoriales invariantes; modelo $6=$ residuos de medida invariantes.

Fuente: Elaboración propia 
teórico-prácticos, como son Matemáticas, Lengua Castellana y Literatura, y Educación Física. Por este motivo, se constató la validez del modelo, primero de manera independiente en cada una de ellas, enfrentándolo, en segundo lugar, con modelos rivales, dado que no se había comprobado en la teoría la existencia de un modelo adecuado (Byrne, 2008). De todos los modelos testados el que presenta mejores valores, en los tres casos, es el que se ha hipotetizado en este estudio, con resultados muy similares, en todos los parámetros estimados, a los observados en el análisis preliminar (AFC). No obstante, para generalizar y poner a prueba de forma más estricta el modelo, se analizó la invarianza factorial. El análisis multistep de invarianza mostró que los modelos presentaban índices de ajuste aceptables.

En todos los casos las variables latentes exógenas o factores contextuales del aprendizaje mostraron niveles de covarianza altos, que oscilaron entre 0.55 y 0.78 , las correlaciones oscilaron entre 0.50 y 0.71 . Creemos que, por un lado, representan niveles de eficiencia de la acción docente y, por otro, que en la práctica la llamada "pedagogía del esfuerzo" y la "pedagogía del interés" se complementan: los alumnos que observaron que los profesores que intentaban promover una cultura del esfuerzo en las clases eran los mismos que intentaban despertar su interés por la materia y viceversa. Además, estás variables se relacionaban positivamente con la acción docente destinada a ayudar a los alumnos a aprender. En la práctica, el profesor eficaz para los alumnos es aquel capaz de despertar el interés por la materia, de ayudarles a aprender enseñando estrategias de apoyo al aprendizaje y que de manera complementaria promueve, valora y reconoce el esfuerzo. Mientras que el mal profesor se caracteriza justamente por lo contrario.

Sin embargo, la actividad cotidiana que el aprendiz percibe en la escuela no es así. Los profesores en todas las materias analizadas promueven más una cultura del esfuerzo que una acción docente destinada a despertar el interés por la materia. En Matemáticas y en Lengua Castellana y Literatura los valores en esta variable están por debajo de la media estadística. No obstante, el valor más alto en las tres variables contextuales y en las tres materias analizadas es la ayuda al aprendizaje.
Los resultados mostraron también que estos factores contextuales predicen, a su vez, factores disposicionales. La acción docente destinada a despertar el interés del alumnado por la materia, realizando propuestas divertidas, diseñando prácticas agradables y brindando una oferta de actividades interesantes y sugestivas predijo significativamente el interés del alumnado por el aprendizaje (en los tres casos el valor predictivo fue el más alto, oscilando entre $\beta=0.79$ y $\beta=0.81$ ). Así mismo, la acción educativa dirigida a promover una cultura del esfuerzo en las clases, valorando, reconociendo y premiando el esfuerzo del alumno se relacionaba significativamente con el esfuerzo informado por los alumnos en el aprendizaje. En este caso, el valor predictivo fue menor, oscilando entre $\beta=$ 0.20 y $\beta=0.23$. Esto es debido, en parte, a que la variable que tiene una mayor influencia sobre el esfuerzo es, como veremos, el interés del alumno por la materia. Por último, la acción del profesor dirigida a guiar al alumno, a enseñarle a aprender, a ayudarle cuando tiene problemas de aprendizaje, predice significativamente la progresión del alumno en el aprendizaje, los valores oscilan entre $\beta=$ 0.12 y $\beta=0.23$. Estos resultados muestran que la acción docente (variables contextuales) repercute directamente en la actitud y aptitud del alumno (variables disposicionales).

Los resultados también mostraron que el interés y el esfuerzo del alumno predicen significativamente el aprendizaje. En Matemáticas, el valor predictivo del esfuerzo es mayor, y en Educación Física y Lengua Castellana y Literatura lo es el interés por el aprendizaje. No obstante, a esta relación directa tenemos que añadir una relación indirecta del interés sobre la progresión por el aprendizaje a través del esfuerzo, en todos los casos significativa y en Educación Física con un valor $\beta=0.53$. Es decir, que el interés y el esfuerzo son variables, como se ha hipotetizado, que: a) inciden por separado en el aprendizaje y, por tanto, se complementan, y b) interaccionan entre sí en el proceso de aprender y, por tanto, interactúan. Hay alumnos que llegan al aprendizaje fundamentalmente a través del esfuerzo, en buena medida como consecuencia de construir en las clases esta cultura (el mayor valor 
predictivo se observa en las clases de matemáticas, $\beta=0.44)$. Otros llegan principalmente a través del interés por aprender, en buena medida, como consecuencia de la acción docente destinada a promover el interés por la materia (el mayor valor predictivo se observa en las clases de Lenguas castellana y Literatura, $\beta=0.35$ ). Y otros llegan a través de un esfuerzo, que es consecuencia de divertirse aprendiendo, o de un esfuerzo que intermedia entre el interés por la materia y los resultados del aprendizaje (el mayor valor predictivo se observa en las clases de Educación Física, $\beta=0.53$ ).

Entre las variables disposicionales, la que tiene un mayor peso estadístico en las tres materias es el esfuerzo invertido para aprender y la que tiene una menor presencia es el interés por la materia, salvo en Educación Física. En Matemáticas y Lengua castellana y Literatura están por debajo de la media estadística. Estos resultados son consistentes con los observados en otros estudios (Hektner et al., 2007; Shernoff et al., 2003).

Con base en estos resultados, creemos que se debe promover una acción docente destinada a despertar el interés del alumno por la materia, porque sabemos que incide directamente en los niveles informados de interés e indirectamente en los de esfuerzo y progresión en el aprendizaje. En la actualidad es la variable contextual la que tiene una menor presencia en las aulas (tabla 1). También se debe promover una cultura del esfuerzo. Construir esta cultura es uno de los grandes retos de la pedagogía actual y debería ser incluido en el sistema educativo como un contenido actitudinal específico (Vinuesa, 2002).

De las tres variables contextuales, la que tiene un mayor peso estadístico es la ayuda al estudiante. Es necesario desplazar el centro de interés desde el sujeto que enseña al sujeto que aprende (Dunkin, 1995), es decir, pensar la enseñanza desde el aprendizaje (Fernández, 2008). En este nuevo modelo se destaca la necesidad de poner el acento en la implicación personal y el compromiso de la persona que aprende en su propio aprendizaje (Núñez, Solano, González-Pienda, \& Rosario, 2006). Esto obliga al profesor a reformular su acción docente y promover un cambio metodológico destinado no ha transmitir de una manera casi exclusiva conocimientos, sino situándose en un contexto mucho más amplio, a guiar al alumno en su proceso de aprendizaje (Cecchini et al., 2013). Los alumnos perciben, en las tres materias, un profesor más dispuesto a promover una cultura del esfuerzo que a despertar su interés por el aprendizaje. Como se ha comentado, despertar el interés del alumno por la materia se sitúa en Matemáticas y en Lengua Castellana y Literatura por debajo de la media estadística, por lo que observamos una cierta descompensación que debería ser corregida.

Por lo que respecta a la relación entre esfuerzo e interés, resulta significativo que la variable que tiene un mayor valor predictivo sobre el esfuerzo no sea la cultura del esfuerzo desplegada por el profesor en sus clases, sino el interés que en los alumnos despierta la materia. Estos resultados se observan en las tres materias analizadas, si bien en Educación Física el valor predictivo es mayor. El esfuerzo, en este caso, es la consecuencia de la implicación personal hacia una actividad que resulta placentera y lo es porque nos permite desplegarnos en ella de manera autónoma, interaccionando con los otros en la búsqueda de un objetivo común y sintiéndonos competentes. En definitiva, si queremos que los alumnos se esfuercen, el mejor método es el de despertar su interés por los contenidos de aprendizaje.

Este estudio tiene algunas limitaciones que deberían ser paliadas en investigaciones posteriores. Se tendrían que realizar nuevos estudios que incluyeran nuevas materias para comprobar la extensión de la invarianza encontrada. También, se deberían comprobar estos resultados en otras etapas educativas. Por último, creemos necesario realizar investigaciones que desarrollen estrategias docentes en cada una de esas dimensiones contextuales del aprendizaje y evalúen sus resultados en programas de intervención en diferentes etapas y materias formativas.

\section{Referencias}

Abalo, J., Lévy, J., Rial, A., \& Varela, J. (2006). Invarianza factorial con muestras múltiples. En J. Lévy (Ed.), Modelización con Estructuras de Covarian- 
zas en Ciencias Sociales (pp. 259-278). Madrid: Netbiblo.

Anderson, J. C., \& Gerbing, D. W. (1988). Structural equation modeling in practice: A review and recommended two-step approach. Psychological Bulletin, 103(3), 411-423.

Bentler, P. M. (2006). EQS structural equations program manual. Encino, CA: Multivariate Software.

Byrne, B. M. (2008). Testing for multigroup equivalence of a measuring instrument: A walk through the process. Psicothema, 20, 872-882.

Carroza, M., Alcaide, A., Heredia, M. F., Humanes, M. A., \& Velasco, F. (2008). El juego de simulación: diseño, desarrollo y evaluación de una actividad para enseñar economía en bachillerato. Iber. Didáctica de las Ciencias Sociales, Geografía e Historia, 58, 48-56.

Cecchini, J. A., Fernández-Losa, J. L., \& Cecchini, C. (2013). La cultura del esfuerzo en las clases de educación física. Cultura y Educación, 25(4), 523-534.

Cecchini, J. A., González-Pienda, J. A., Méndez-Giménez, A., Fernandez-Río, J., González-Losa, J., \& González-Mesa, C. (2014). Propiedades psicométricas de los cuestionarios IEPA y AYES: dos medidas de las variables disposicionales y contextuales para el aprendizaje en Secundaria. Psicothema, 26(1), 76-83.

Cecchini, J. A., Méndez-Giménez, A., \& Fernández-Río, J. (2014). Análisis de un modelo integrador del aprendizaje: relaciones entre variables contextuales y meta-creencias del alumnado de secundaria. Aula Abierta, 42, 90-97.

Centelles, J. (2012). La bandera del Nepal no es rectangular. Aula de innovación educativa, 209, 77-86.

Cheung, G. W., \& Rensvold, R. B. (2002). Evaluating goodness-of-fit indexes for testing measurement invariance. Structural Equation Modeling, 9(2), 233-255.

Colectivo Lorenzo Luzuriaga (2012). Documento sobre el fracaso escolar en el estado de las Autonomías. Barcelona: Wolters Kluwer. Educación

Cuenca, J. M. (2010). La resolución de problemas en la enseñanza de las ciencias sociales a través de videojuegos. Iber. Didáctica de las Ciencias Sociales, Geografía e Historia, 63, 32-42.
Del Burgo, M. (2002). El fracaso escolar. Madrid: Acento Editorial.

Dunkin, M. (1995). Concepts of teaching and teaching excellence in Higher Education. Higher Education Research and Development, 14(1), 21-33.

Enkvist, I. (2012). La buena y la mala educación. Madrid: Encuentro.

Fernández, J. (2008). Valoración de la calidad docente. Madrid: Editorial Complutense.

Fernández, M., Mena, L., \& Riviere, J. (2010). Fracaso y abandono escolar en España. Barcelona: Obra Social Fundación "La Caixa".

Flores, S. (2011). Rock Band en el aula de música. Eufonía: Didáctica de la música, 52, 35-43.

Franco, A. F. (2012). Los elementos químicos y la enseñanza bilingüe de las ciencias. Alambique: Didáctica de las ciencias experimentales, 71, 99-103.

Häusemann, G. (2011). La enseñanza de la física a través de los juguetes. Alambique: Didáctica de las ciencias experimentales, 67, 79-87.

Hektner, J. M., Schmidt, J. A., \& Csikszentmihalyi, M. (2007). Experience sampling method. Measuring the quality of everyday life. California: Sage Publications.

Hu, L., \& Bentler, P. M. (1999). Cutoff criteria for fit indexes in covariance structure analysis: Conventional criteria versus new alternatives. Structural Equation Modeling, 6, 1-55.

Larson, R. W., \& Richards, M. H. (1991). Boredom in the Middle School years: Blaming schools versus blaming students. American Journal of Education, 99(4), 418-443.

Lévy J.P., Martín M.T., \& Román M.V. (2006). Optimización según estructuras de covarianzas. En J. P. Lévy, \& J. Varela (Eds.), Modelización con estructuras de covarianzas en ciencias sociales (pp. 11-30). Coruña: Netbiblo.

Lozano, A. (2003). Factores personales, familiares, y académicos, que afectan el fracaso escolar en la educación secundaria. Electronic Journal of Research in Educational Psychology, 1(1), 43-66.

Marchesi, A. (2003). El fracaso escolar en España. Madrid: Fundación Alternativas.

Marsh, H. W. (1993). The multidimensional structure of physical fitness: Invariance over gender and 
age. Research Quarterly for Exercise and Sport, 64, 256-273.

Méndez-Giménez, A. (2011) (Coord.). Modelos actuales de iniciación deportiva escolar. Unidades didácticas sobre juegos y deportes de cancha dividida. Sevilla: Ed. Wanceulen.

Mendoza, L. M. (2008). Una experiencia en la educación matemática. Uno: Revista de didáctica de las matemáticas, 47, 106-11.

Nunnally, J. C. (1978). Psychometric theory. New York: McGraw-Hill.

Núñez, J. C., Solano, P., González-Pienda, J. A., \& Rosario, P. (2006). Evaluación de los procesos de autorregulación mediante autoinforme. Psicothema, 18(3), 353-358.

Roca, E., Carmona, J., Boix, C., Colomé, R., López, A., Sanguinetti, A., Caro, M., \& Sans Fitó, A. (2010). El aprendizaje en la infancia y en la adolescencia. Claves para evitar el fracaso escolar. Esplugues de Llobregat: Hospital Sant Joan de Déu.

Sabater, LL. (2009). Tres juegos para practicar las Matemáticas casi sin darse cuenta. Uno: Revista de didáctica de las matemáticas, 50, 108-117.

Satorra, A., \& Bentler, P. M. (1994). Corrections to test statistics and standard errors in covariance structure analysis. En A. von Eye, \& C.C. Clogg (Eds.): Latent variables analysis: Applications for developmental research (pp. 399-419). Thousand Oaks, CA: Sage.
Schank, R. C. (2005). Lessons in Learning, e-Learning, and Training: Perspectives and Guidance for the Enlightened Trainer. New York: Wiley and Sons.

Schiefele, U., \& Csikszentmihalyi, M. (1995). Motivation and ability as factors in mathematics experience and achievement. Journal for Research in Mathematics Education, 26(2), 163-181.

Shernoff, D. J., Csikszentmihalyi, M., Schneider, B., $\&$ Shernoff, E. S. (2003). Student engagement in High school classrooms from the perspective of Flow Theory. School Psychology Quarterly, 18(2), 158-176.

Steiger, J. H. (1990). Structural model evaluation and modification: An interval estimation approach. Multivariate Behavioral Research, 25, 173-180.

Trilla, J. (2002) La aborrecida escuela. Junto a una pedagogía de la felicidad y otras cosas. Barcelona: Laertes.

Unesco (2012). Informe de Seguimiento de la EPT en el Mundo. Los jóvenes y las competencias: trabajar con la educación. París: Organización de las Naciones Unidas para la Educación, la Ciencia y la Cultura. Vinuesa, M. (2002). Construir los valores. Curriculum con aprendizaje cooperativo. Bilbao: Desclée de Brouwer.

Wong, M. M-H. (2000). The relations among causality orientations, academic experience, academic performance, and academic commitment. Personality and Social Psychology Bulletin, 26, 315-326.

Yair, G. (2000). Reforming motivation: How the structure of instruction affects students learning experiences. British Educational Research Journal, 26(2), 191-210. 
